\title{
NUCLEAR STAR CLUSTERS FROM CLUSTERED STAR FORMATION
}

\author{
Meghann Agarwal ${ }^{1}$ And Miloš Milosavluević ${ }^{2}$ \\ ${ }^{1}$ Department of Physics, University of Texas, 1 University Station C1600, Austin, TX 78712, USA \\ ${ }^{2}$ Department of Astronomy and Texas Cosmology Center, University of Texas, 1 University Station C1400, Austin, TX 78712, USA \\ Received 2010 August 12; accepted 2011 January 1; published 2011 February 7
}

\begin{abstract}
Photometrically distinct nuclear star clusters (NSCs) are common in late-type disk and spheroidal galaxies. The formation of NSCs is inevitable in the context of normal star formation in which a majority of stars form in clusters. A young, mass-losing cluster embedded in an isolated star-forming galaxy remains gravitationally bound over a period determined by its initial mass and the galactic tidal field. The cluster migrates radially toward the center of the galaxy and becomes integrated in the NSC if it reaches the center. The rate at which the NSC grows by accreting young clusters can be estimated from empirical cluster formation rates and dissolution times. We model cluster migration and dissolution and find that the NSCs in late-type disks and in spheroidals could have assembled from migrating clusters. The resulting stellar nucleus contains a small fraction of the stellar mass of the galaxy; this fraction is sensitive to the high-mass truncation of the initial cluster mass function (ICMF). The resulting NSC masses are consistent with the observed values, but generically, the final NSCs are surrounded by a spatially more extended excess over the inward-extrapolated exponential (or Sérsic) law of the outer galaxy. We suggest that the excess can be related to the pseudobulge phenomenon in disks, though perhaps not all of the pseudobulge mass assembles this way. Comparison with observed NSC masses can be used to constrain the truncation mass scale of the ICMF and the fraction of clusters suffering prompt dissolution. We infer truncation mass scales of $\lesssim 10^{6} M_{\odot}$ $\left(\gtrsim 10^{5} M_{\odot}\right.$ ) without (with 90\%) prompt dissolution. Since the NSC assembly is collisionless and non-dissipative, no relation to the process responsible for central black hole assembly in more massive galaxies is expected.
\end{abstract}

Key words: galaxies: evolution - galaxies: kinematics and dynamics - galaxies: nuclei - galaxies: star clusters: general - galaxies: structure

\section{INTRODUCTION}

Photometrically distinct nuclear star clusters (NSCs) are ubiquitous in dynamically primitive galaxies, which are the galaxies that lack what can be interpreted as the structural signatures of major-"wet" or "dry"-mergers: surface brightness profiles with de Vaucouleurs-like or higher Sérsic (1963) indices, and, in the absence of core scouring by binary massive black holes, power-law central luminosity cusps (e.g., Hopkins et al. 2008, 2009a, 2009b, and references therein). Imaging with the Hubble Space Telescope (HST) has revealed that 75\% of late-type ( $\mathrm{Sc}-\mathrm{Sd}$ ) disk galaxies contain compact, luminous star clusters at their centers with masses in the range $8 \times 10^{5}$ to $6 \times 10^{7} M_{\odot}$, effective radii in the range $1-9 \mathrm{pc}$ with a median of $\sim 3.5 \mathrm{pc}$, and velocity dispersions in the range $13-34 \mathrm{~km} \mathrm{~s}^{-1}$ (e.g., Böker et al. 2002, 2004; Walcher et al. 2005). The clusters' ages range widely from $4 \times 10^{7}$ to $10^{10} \mathrm{yr}$, the metallicities average at $\langle Z\rangle=0.015$ with a significant scatter, the average star formation rate over the last $100 \mathrm{Myr}$ is $\left\langle\Sigma_{\mathrm{SFR}}\right\rangle=2 \times 10^{-3} M_{\odot} \mathrm{yr}^{-1}$, and older stars dominate the cluster mass. Similarly, an HST survey of stellar nuclei in spheroidal galaxies in the Virgo and Fornax Clusters (Ferrarese et al. 2006a; Côté et al. 2006; Miller \& Lotz 2007) has revealed stellar nuclei that on average contain $\sim 0.2 \%$ of the galaxy mass (Ferrarese et al. 2006b; Wehner \& Harris 2006) and have a median effective radius of $4.2 \mathrm{pc}$ (see also Geha et al. 2002). Also, HST imaging of nearby low-luminosity dwarf galaxies with absolute magnitudes $-16<M_{V}<-13$ (Georgiev et al. 2009) has revealed NSCs with masses $\sim\left(10^{6}-10^{7}\right) M_{\odot}$, with a majority clustering at the lower end of the mass range, and half-light radii $1.5-4.7 \mathrm{pc}$.

It seems that photometrically distinct NSCs are common in galaxies that have not experienced major mergers in the epoch following the initial burst of star formation (see, e.g., Koda et al. 2009), but have a well-defined dynamical center. The faintest dwarf spheroidal satellites of the Milky Way, with the exception of the Sagittarius Dwarf (e.g., Bellazzini et al. 2008, and references therein), do not contain NSCs, even though they do seem to form a continuous structural family of stellar systems with the more massive dwarf spheroidals that do contain NSCs (e.g., Kormendy et al. 2009; Wyse 2010, and references therein). While the spheroidal galaxies in Virgo are typically non-rotating (Geha et al. 2003), their nucleus-subtracted surface brightness profiles, with Sérsic indices $n \sim 1-2$, resemble those of disks. This motivates a unified approach to addressing NSC formation in these two morphological classes. In disks, an excess surface brightness above the inward-extrapolated exponential law of the outer disk occurring at radii $\sim 100-500 \mathrm{pc}$, variously characterized as "central light excess" (Böker et al. 2003) or a "pseudobulge" (Kormendy \& Kennicutt 2004; Fisher \& Drory 2008, 2010; Fisher et al. 2009; Weinzirl et al. 2009), may provide additional clues, because the process that produced the NSC could also contribute to the formation of the pseudobulge. Pseudobulges can be distinguished from classical bulges through their cold, rapidly rotating kinematics and low Sérsic index (see Kormendy \& Fisher 2008, and references therein). Kormendy et al. (2010) find that the pseudobulges in the sample of pure-disk (Scd) galaxies contain $\lesssim 3 \%$ of the galactic stellar mass, which is smaller than the stellar mass fraction in classical bulges.

Here we discuss the formation of NSCs in the context of normal star formation in which most stars form in clusters. A successful theory of NSC formation should explain the observed uniformity of their properties. The smallness of their masses in comparison with the total stellar masses of the host galaxies is a "small parameter" characterizing the assembly of cosmic structure that requires explanation. A theory of NSC formation 
could help constrain the earliest stages of the host galaxy's formation. NSCs are interesting because they seem to betray either an inefficiency in the transport of baryons (gas, stars) to the center of the galaxy, or perhaps an inefficiency of star formation once gas has arrived at the center of the galaxy. The NSC phenomenon may be related to the relatively slow, or "secular" buildup of the host galaxy's stellar mass, since the galaxies that form the bulk of their stars on dynamical timescales, as in mergers (e.g., Hopkins et al. 2008, 2009a) and cold accretion from the intergalactic environment (e.g., Dekel et al. 2009b; Ceverino et al. 2010), should turn out to be more concentrated than the NSC host galaxies and should contain "classical" bulges structurally equivalent with ellipticals.

NSCs can grow from the local interstellar medium if the galactic gas accretes and accumulates at the center (e.g., Seth et al. 2006). In gas disks, sufficient gas accretion to form NSCs may simply be driven by magnetic stresses in the gas that are amplified by the magnetorotational instability (Milosavljević 2004), which is generic in differentially rotating galactic disks (e.g., Kim et al. 2003). If the inner baryonic disk is globally self-gravitating, accretion can be driven by torques associated with stellar and gaseous bars (e.g., Kormendy \& Kennicutt 2004; Schinnerer et al. 2007, and references therein). NSCs in late-type disks typically contain young stellar components, and their star formation seems to be intermittent (Rossa et al. 2006; Walcher et al. 2006), which is consistent with the gas accretion scenario. Cosmological hydrodynamical simulations of dwarf and latetype galaxy formation commonly yield nucleus- (or "bulge-") disk structures (e.g., Governato et al. 2004, 2007, 2009; Kaufmann et al. 2007; Brooks et al. 2009; Ceverino et al. 2010), where the nucleus forms from the gas that self-gravitational torquing has driven into the center of the galaxy, whereas the disk forms from the gas that manages to stably circularize in the combined gravitational potential of the dark matter halo and gaseous stellar nucleus. The resulting nuclei normally contain larger fractions of galaxy mass than the observed nuclei in late-type disks and spheroidals. Frequently, a gas expulsion by feedback from star formation is invoked to explain this apparent discrepancy (e.g., Zavala et al. 2008; Dutton 2009).

Here we do not study gas accretion, but instead investigate the possibility, which is necessitated by the clustered character of star formation, that a substantial fraction of the NSC mass could have assembled in off-center stellar associations (clusters) that subsequently migrated into the galactic centers and merged into an NSC as intact entities (e.g., Tremaine et al. 1975; Lotz et al. 2001; Capuzzo-Dolcetta \& Miocchi 2008a, 2008b). The assembly of NSCs through the merging of clusters is consistent with the observation that nuclear cluster phase space densities are on average somewhat smaller than those of globular clusters (Walcher et al. 2005) because phase space density tends to increase in collisionless mergers. The NSCs thus assembled should inherit the orbital angular momentum of the migrating disk clusters and should be rotating (see, e.g., Seth et al. 2008).

Bekki (2010) carried out $N$-body simulations of star cluster orbital decay in the background of field stars in a disk galaxy embedded in a dark matter halo. His results suggest that NSCs could have formed from stars delivered by inspiraling star clusters. In a similar spirit but different context and following an earlier proposal by Noguchi (1999), Immeli et al. (2004), Bournaud et al. (2007), Elmegreen et al. (2008b), and Ceverino et al. (2010) carried out numerical simulations to find that the migration and central merging of giant star clusters and gravitationally bound gas clumps in massive, rapidly star- forming disks can give rise to classical-bulge-like, dynamically hot central stellar systems. If giant cluster migration can produce classical bulges in gravitationally unstable disks that are fed by a relatively smooth accretion from the extragalactic environment-and not by major mergers (see, e.g., Genzel et al. 2008) - then this warrants an investigation of cluster migration in lower surface density galaxies, in the hope that an improved understanding of their morphological transformation can be gained to elucidate why these galaxies end up lacking classical bulges and remain rotationally dominated and dynamically cold.

To this end, we develop a crude toy model to calculate the influx of migrating young clusters formed in a galaxy that has experienced an instantaneous star formation episode, but our results are relevant for galaxies, like the prototypical late-type disk galaxy M33, in which star formation has been steady and ongoing over the life of the galaxy, although the timescale for the accumulation of NSC mass will differ from the one estimated here because of the dependence, in this case, on the star formation rate in the disk. We assume that all stars form in clusters and that the clusters eventually get disrupted in the galactic tidal field. We further discuss the assumptions and limitations of our model in Section 3.4. Gas expulsion, stellar mass loss, dynamical evaporation, and external perturbations lead to either rapid or progressive tidal mass loss in the clusters (e.g., Fall \& Zhang 2001; Baumgardt \& Makino 2003; McMillan \& Portegies Zwart 2003; Gieles et al. 2006c). If a cluster is massive, it can migrate radially inward prior to complete disruption. For example, we will find that for our galactic models and parameters (see Sections 3 and 4 ) a $10^{4} M_{\odot}$ cluster never migrates more than $10 \mathrm{pc}$; a $10^{5} M_{\odot}$ cluster can migrate and reach the center from $\sim$ few $\times 10 \mathrm{pc}$, a $10^{6} M_{\odot}$ cluster can do so from $\sim 100 \mathrm{pc}$, and a $10^{7} M_{\odot}$ cluster can reach the center from $\gtrsim 1 \mathrm{kpc}$. The cluster's stars are then deposited at galactocentric radii traversed by the migrating cluster. Since even in very late type disks (Hubble-type Scd) circular test particle orbits rotate differentially throughout, cluster disruption is inevitable except if the cluster forms at, or migrates into, the galactic center, where tidal shear preserves the symmetries of an axisymmetric cluster. Clusters with off-center birthplaces can avoid complete disruption if they migrate to the dynamical center quickly enough. The progressive tidal disruption of migrating clusters before they have reached the galactic center transports mass radially inward; we propose that this process is a driver of pseudobulge growth in disks.

This work is organized as follows. In Section 2, we provide an empirically and theoretically motivated description of cluster formation, migration, and dissolution. In Section 3, we introduce our models for a spheroidal galaxy and a disk galaxy initially lacking an NSC and estimate the effects of galactic stellar mass redistribution due to cluster migration. In Section 4, we show our results for representative galaxy models. In Section 5, we compare our results with observations of NSCs and pseudobulges and provide a brief discussion of implications for the pseudobulge and classical bulge dichotomy and for massive black holes, and in Section 6 we summarize our main conclusions.

\section{CLUSTER FORMATION, MIGRATION, AND DISSOLUTION}

We assume that all stars in our model galaxies formed in clusters, and introduce a framework based on previous empirical 
and theoretical work for treating the formation, migration, and dissolution of these young stellar clusters. In Section 2.2, we discuss a theoretically motivated description of cluster migration in spheroidal and disk galaxies, taking into account the dominant form of dynamical friction torque acting on a migrating cluster in each type of galaxy. In Section 2.3, we describe an empirically motivated model of cluster disruption based on observations of young clusters in disk galaxies. In Section 2.1, we introduce the initial cluster mass function (ICMF), which seems to be generic to star formation in all galaxies, including the Milky Way.

\subsection{The Initial Cluster Mass Function}

The ICMF contains about equal mass on all cluster mass scales, i.e.,

$$
\frac{d n}{d M} \propto M^{-\alpha} \text { for } M_{\min }<M<M_{\max },
$$

with $\alpha \sim 2$ (e.g., Bik et al. 2003; de Grijs et al. 2003; Hunter et al. 2003; Rafelski \& Zaritsky 2005; Chandar et al. 2010), where $M$ denotes initial cluster mass and $M_{\min }$ and $M_{\max }$ are galaxy-dependent cutoffs. We adopt $\alpha=2$ and $M_{\min }=100 M_{\odot}$ in what follows; our results will depend only weakly on $M_{\min }$ since clusters with masses below $\sim 10^{4} M_{\odot}$ are disrupted before they can migrate to the center from larger radii and, if they do reach the center, contribute an insignificant fraction of the total NSC mass. The definition of a high-mass cutoff of the ICMF and the statistical significance of observational evidence for such a cutoff have been the subject of debate. We assume here that the ICMF is indeed subject to high-mass truncation and treat $M_{\max }$ as a free parameter that varies over the range $10^{4} M_{\odot}-10^{7} M_{\odot}$, which includes the cutoffs reported for the nearby galaxies. We will find in Section 4 that masses of nuclear clusters in our calculations are most sensitive to the truncation mass, and thus the photometry, of spheroidal and disk galaxies. If combined with a theoretical model of cluster migration and dissolution, this sensitivity can be utilized to indirectly constrain the ICMF truncation mass scale in these galaxies.

The maximum cluster mass forming in NGC 6946, M51, and the Antennae has been estimated from observations to be $M_{\max } \sim 10^{6} M_{\odot}$ (Gieles et al. 2006a, 2006b). Frequently, an ICMF with an exponential cutoff $d n / d M \propto M^{-\alpha} e^{-M / M_{\star}}$ is found to adequately approximate the truncation of the ICMF at the high-mass end. In spirals and irregulars, the mass truncation scales $M_{\star}$ and $M_{\max }$ are in the range $\sim 2 \times 10^{5}$ to $10^{7.5} M_{\odot}$ (see, e.g., Zhang \& Fall 1999; Bik et al. 2003; de Grijs et al. 2003; Dowell et al. 2008; Larsen 2009; Gieles 2009). The exponential mass truncation scale, which has typical values of $M_{\star} \sim 2 \times 10^{5} M_{\odot}$, is larger in denser starburst environments (Larsen 2009). In the present work, we adopt the abrupt truncation with $M_{\max }$, but we do not anticipate that the results would be substantially different if exponential truncation were used.

The maximum mass of clusters that formed in the assembly of nucleated spheroidals is unknown, but a brief theoretical speculation may be in order. In a gas disk with surface density $\Sigma_{\text {gas }}$ close to the critical value $\sim 10 M_{\odot} \mathrm{pc}^{-2}$ corresponding to the column density $N \sim 10^{21} \mathrm{~cm}^{-2}$ that is required for the presence of a self-shielding cold phase (see, e.g., Schaye 2004) and Toomre parameters $Q \lesssim 1$, the truncation scale $M_{\max }$ (or $M_{\star}$ ) should scale with the Jeans mass of the disk,

$$
\begin{aligned}
M_{\mathrm{max}} & \sim f_{\mathrm{SF}}\left(\frac{4 \pi^{2} G \Sigma_{\mathrm{gas}}}{\kappa^{2}}\right)^{2} \Sigma_{\mathrm{gas}} \\
& \sim 4 \times 10^{6} M_{\odot} f_{\mathrm{SF},-1}\left(\frac{\Sigma_{\mathrm{gas}}}{10 M_{\odot} \mathrm{pc}^{-2}}\right)^{3}\left(\frac{\kappa}{10^{-15} \mathrm{~s}^{-1}}\right)^{-4},
\end{aligned}
$$

where $f_{\mathrm{SF}}=0.1 f_{\mathrm{SF},-1}$ is the star formation efficiency and $\kappa$ is the epicyclic frequency (see, e.g., Elmegreen et al. 2008b). The reference epicyclic frequency in Equation (2) was selected to correspond to the characteristic average total mass density $\sim 0.1 M_{\odot} \mathrm{pc}^{-3}$ in the inner $300 \mathrm{pc}$ of the (largely non-nucleated) dwarf spheroidal satellites of the Milky Way (Strigari et al. 2008), but of course, the proto-spheroidals would have been characterized by larger, radius-dependent values of $\kappa$. If the gas disk mass is a fixed proportion of the total (e.g., dark matter) enclosed mass, and the vertical scale height of the disk is $h$, then $\kappa \propto \Sigma_{\text {gas }}^{1 / 2} h^{-1 / 2}$, which would imply a weaker dependence $M_{\text {max }} \propto \Sigma_{\text {gas }} h^{2}$ on the gas surface density. It is plausible that proto-spheroidals assembled from an ICMF reaching the cluster mass scale associated with the gas disk Jeans mass estimated in Equation (2).

\subsection{Cluster Migration}

A gravitationally bound star cluster migrates on a timescale

$$
t_{\mathrm{mig}}=\frac{d J}{d \ln R}\left\langle T_{\mathrm{DF}}\right\rangle^{-1},
$$

where $R$ is a characteristic size of the orbit-the equivalent of the semimajor axis in a non-Keplerian potential, $J$ is the angular momentum of the cluster, and $\left\langle T_{\mathrm{DF}}\right\rangle$ is the orbit-averaged dynamical friction torque. All galactic mass components (dark matter, gas disk, and stars) respond dynamically to the cluster but the mechanism of torque coupling varies. We separately consider the dynamical friction torque in spheroidal and disky mass components.

In spheroidal stellar systems or dark matter halos, $N$-body simulations have shown that the torque from a non-rotating collisionless halo can be heuristically described with the Chandrasekhar formula for dynamical friction,

$$
\vec{T}_{\mathrm{DF}, \text { Chandra }}=\frac{4 \pi \ln (\Lambda) \chi(V) G^{2} M^{2} \rho}{V^{3}} \vec{r} \times \vec{V},
$$

where $\rho$ is the local combined density of stars and dark matter, $|\vec{V}| \sim \Omega R$ is the velocity of the cluster, $\ln (\Lambda)$ is the Coulomb logarithm, and $\chi(V)$ is the mass fraction of stars or dark matter particles with velocities less than $V$. Because the kinematic structure of actual halos differs from the premises of Chandrasekhar's derivation, $N$-body simulations are necessary to obtain the correct normalization of the torque amplitude. The numerically evaluated torques (e.g., Velazquez \& White 1999; Peñarrubia et al. 2002, 2004; Spinnato et al. 2003) can be modeled with Chandrasekhar's formula if the Coulomb logarithm is treated as an empirically calibrated free parameter. The values of the Coulomb logarithm obtained from these $N$ body simulations are $\ln (\Lambda) \sim 2-7$.

In differentially rotating gas or stellar disks, the torque is provided by the flow in the corotation region and by angular momentum transfer at Lindblad resonances (e.g., Goldreich 
\& Tremaine 1980; Quinn \& Goodman 1986). Disk clusters with masses $M \lesssim 10^{6} M_{\odot}$ have Roche tidal radii $r_{\mathrm{t}} \sim$ $\left[G M /(d \Omega / d \ln R)^{2}\right]^{1 / 3}$ that are smaller than the thickness of the disk. In this regime, the disk torque is a generalization of the "Type I" torque acting on small planets in protoplanetary disks (e.g., Tanaka et al. 2002; D’Angelo et al. 2003; Baruteau $\&$ Masset 2008) to non-Keplerian disks and is proportional to

$$
T_{\mathrm{DF}, \text { disk }} \propto \frac{G^{2} M^{2} \Sigma}{\sigma^{2}},
$$

where $\Sigma$ is the surface density of the gas or stellar disk and $\sigma$ is the gas sound speed or the stellar velocity dispersion in the disk. In what follows, we take the constant of proportionality in Equation (5) to be equal to unity. This choice seems consistent with the numerical calibration of the torque in a collisionless particle disk by Donner \& Sundelius (1993) and Wahde et al. (1996) if the gravitational softening employed in their calculations is equated with the vertical thickness of the disk.

Finally, we note that Milosavljević (2004) argued against the migratory scenario in disks on the grounds of long migration times. There, migration from a distant location in the disk was envisioned, and the treatment ignored the enhancement in dynamical friction force due to the stellar accumulation of earlier migrating clusters in the central kiloparsec of the galaxy. In the present picture, clusters migrate from a range of radii in the galactic disk, but only those that form closest to the galactic center reach the center and merge with the nuclear cluster.

\subsection{Cluster Dissolution}

The timescale on which a cluster is disrupted in the galactic tidal field has been determined empirically by modeling the luminosity and age functions of clusters in nearby disk galaxies (see, e.g., Lamers et al. 2005a, 2005b). Theoretical models tracking stellar and dynamical evolution of a cluster in a tidal field (e.g., Gieles et al. 2008; Gieles \& Baumgardt 2008) have reproduced the observationally inferred variation of the dissolution time with cluster mass,

$$
t_{\mathrm{dis}}=t_{0}\left(\frac{M}{M_{\odot}}\right)^{\gamma},
$$

where $\gamma \approx 0.62$ (Boutloukos \& Lamers 2003; de Grijs \& Anders 2006). The normalization $t_{0}$ varies between galaxies, which seems to be a consequence of the variation in the tidal field strength and of cluster-scale density inhomogeneities of the galactic environment. Gieles et al. (2008) assessed the role of the tidal field by comparing cluster lifetimes in several galaxies with the inverse angular frequency of circular orbits at observed cluster radii and found consistency with the linear relation $t_{\text {dis }} \propto \Omega^{-1}$. The residual variation of $t_{\text {dis }}$ between galaxies has been ascribed to disruption by giant molecular clouds (Gieles et al. 2006c, and references therein) which we ignore. The tidal radius really depends on the degree of differential rotation, $r_{\mathrm{t}}=\left[G M /(d \Omega / d \ln R)^{2}\right]^{1 / 3}$ (e.g., Quinn \& Goodman 1986), and thus one would expect that $t_{0} \propto|d \Omega / d \ln R|^{-1}$. Lamers et al. (2005b) modeled the observed cluster population in M33 and found, at an arbitrary radius, $t_{0} \approx f_{\text {dis }} / \Omega(R)$ with $f_{\text {dis, } \mathrm{M} 33} \approx 0.16$.

The coefficient $f_{\text {dis }}$, which can be separately inferred from the observed young cluster populations and from theoretical calculations, encapsulates the detailed mass-loss mechanics in a cluster embedded in a galactic tidal field and evolving through internal two-body relaxation. From the theoretical viewpoint, the coefficient depends on the initial stellar mass function, on the initial cluster structure, and on the (possibly timedependent) gravitational potential within and near the cluster. Under idealized assumptions, the coefficient has been estimated with $N$-body simulations (Portegies Zwart et al. 1998, 2002; Baumgardt \& Makino 2003). The theoretical estimate $f_{\text {dis }} \sim 0.3$ in Lamers et al. (2005b), which is based on the results of Baumgardt \& Makino (2003), is larger than the empirical value. The clusters originating in M33's central disk could be denser and more resistant to tidal disruption than those in the sample of Lamers et al. (2005b).

The above relations were obtained for clusters originating in a galactic disk. For lack of an equivalent empirical result for spheroidal starbursts, we assume that these relations hold universally and adopt the crude relation

$$
t_{0}=f_{\text {dis }}\left|\frac{d \Omega}{d \ln r}\right|^{-1},
$$

where we take $f_{\text {dis }}=0.2$. We have compared our simplified form of the dissolution timescale defined in Equations (6) and (7) for a cluster with initial mass $M=10^{5} M_{\odot}$ and angular velocity $\Omega(r)=\left[G M_{\mathrm{gal}, 0}(r) / r^{3}\right]^{1 / 2}$, where $M_{\mathrm{gal}, 0}(r)=$ $M_{\star, 0}(r)+M_{\mathrm{DM}}(r)$ is the total initial galactic mass within a sphere of radius $r$ (see Section 3), with the timescale from Lamers et al. (2005a, 2005b), where $t_{\mathrm{dis}}^{\prime}=t_{4}^{\prime}\left(M / 10^{4} M_{\odot}\right)^{\gamma}$ (Boutloukos \& Lamers 2003), $t_{4}^{\prime}=1.355 \mathrm{yr} \times 10^{4 \gamma} \gamma^{-1}\left(t_{0}^{\prime} / 1 \mathrm{yr}\right)^{0.967}$ (Lamers et al. 2005a), and $t_{0}^{\prime}=f_{\text {dis }} \Omega(r)^{-1}$, and found it to be consistent to within $20 \%$ in the range of radii where we find that significant cluster migration takes place.

\section{INITIAL AND REDISTRIBUTED GALACTIC STELLAR MASS}

Here we describe our models for the initial density profile of a spheroidal galaxy (Section 3.1) and a disk galaxy (Section 3.2). The initial density lacks a stellar nucleus; its innermost baryonic density profile is an extrapolation from larger radii. Then in Section 3.3, we describe our method of distributing initial clusters consistent with these surface density profiles and modeling cluster mass redistribution during migration and dissolution. We include the growing central cluster mass in the mass distribution affecting subsequent migrating clusters, but we ignore the response of the dark matter halo to the baryonic collapse and subsequent mass redistribution by cluster migration.

\subsection{Spheroidal Galaxy Model}

Photometry of spheroidal galaxies in the nearby universe (Côté et al. 2006; Ferrarese et al. 2006a; Kormendy et al. 2009), combined with the fact that in these relatively old stellar systems the stellar mass-to-light ratios do not vary significantly within galaxies, has shown that their mass surface density profiles are well described by a two-component model (Balcells et al. 2003; Graham \& Guzmán 2003)

$$
\Sigma_{\star}(R)=\Sigma_{\text {Sersic }}(R)+\Sigma_{\text {nucl }}(R) .
$$

The Sérsic law component $\Sigma_{\text {Sersic }}(R) \propto \exp \left[-\left(R / R_{\mathrm{S}}\right)^{1 / n}\right]($ Caon et al. 1993), where $n$ is the Sérsic index and $R_{\mathrm{s}}$ is a scale radius, contains most of the mass, and the compact nuclear 
component $\Sigma_{\text {nucl }}(R)$ contains a small fraction (Ferrarese et al. 2006b; Wehner \& Harris 2006) of the mass. In spheroidals, the Sérsic index varies in the range $n \sim 1-2$ (Ferrarese et al. 2006a; Kormendy et al. 2009). We work within the paradigm in which a stellar nucleus is not present prior to the formation of most of the galactic stars. Thus, we consider an initial galaxy with a spherically averaged stellar density profile that lacks a nucleus. We employ an approximation to the deprojected Sérsic profile in the form (Prugniel \& Simien 1997; Lima Neto et al. 1999)

$$
\rho_{\star, 0}(r)=\rho_{\mathrm{sph}}\left(\frac{r}{R_{\mathrm{s}}}\right)^{-p} \exp \left[-\left(\frac{r}{R_{\mathrm{s}}}\right)^{1 / n}\right],
$$

where

$$
p=1-0.6097 n^{-1}+0.05463 n^{-2} \text {. }
$$

Here, $\rho_{\text {sph }}$ is normalized to the total stellar mass of the galaxy $M_{\mathrm{sph}}=4 \pi \int_{0}^{\infty} \rho_{\star, 0}(r) r^{2} d r=4 \pi n R_{\mathrm{s}}^{3} \rho_{\mathrm{sph}} \Gamma[(3-p) n]$, and $\Gamma(a)=\int_{0}^{\infty} t^{a-1} e^{-t} d t$ is the Gamma function. Quantities pertaining to the initial galaxy, before migration and disruption of clusters, are denoted by the subscript 0 . The stellar mass within radius $r$ is

$$
M_{\star, 0}(r)=4 \pi n R_{\mathrm{s}}^{3} \rho_{\mathrm{sph}} \gamma\left[(3-p) n,\left(r / R_{\mathrm{s}}\right)^{1 / n}\right],
$$

where $\gamma(a, x)=\int_{0}^{x} t^{a-1} e^{-t} d t$ is the lower incomplete Gamma function.

The total initial density profile includes a dark matter component

$$
\rho_{0}(r)=\rho_{\star, 0}(r)+\rho_{\mathrm{DM}}(r),
$$

where we model the dark matter density profile with the Navarro-Frenk-White (NFW) law (Navarro et al. 1997) $\rho_{\mathrm{DM}}(r)=\rho_{s} /\left[\left(r / r_{s}\right)\left(1+r / r_{s}\right)^{2}\right]$ with scale radius $r_{\mathrm{s}}$, virial radius $r_{\text {vir }}$, and concentration $c \equiv r_{\text {vir }} / r_{\mathrm{s}}$. Spheroidals are often dark matter dominated, especially at the low-luminosity end, i.e., $\rho_{\mathrm{DM}}(r) \gtrsim \rho_{\star, 0}(r)$. Thus, for galaxies in which dark matter is the dominant mass component, the cluster migration time can be modeled with the $N$-body-simulation-calibrated Chandrasekhar dynamical friction torque in Equation (4).

\subsection{Disk Galaxy Model}

The surface density profiles of late-type spiral disk galaxies can be fit with a Sérsic-type exponential stellar disk and a nuclear component (Böker et al. 2003) as in Equation (8). We consider "pure" disk galaxies, which do not have a stellar bulge or a preexisting pseudobulge; we do not consider cluster formation in the galactic halo. The initial stellar density profile without the nuclear component is then

$$
\Sigma_{\star, 0}(R)=\Sigma_{\text {disk }} \exp \left[-\left(\frac{R}{R_{\mathrm{S}}}\right)^{1 / n}\right],
$$

where $\Sigma_{\text {disk }}=M_{\text {disk }} /\left[2 \pi n R_{\mathrm{s}}^{2} \Gamma(2 n)\right]$. The corresponding initial stellar mass profile is

$$
M_{\star, 0}(R)=2 \pi n R_{\mathrm{s}}^{2} \Sigma_{\mathrm{disk}} \Gamma\left[2 n,\left(R / R_{\mathrm{s}}\right)^{1 / n}\right],
$$

where $\Gamma(a, x)=\Gamma(a)-\gamma(a, x)$ is the upper incomplete Gamma function. The total initial density profile is then

$$
\Sigma_{0}(R)=\Sigma_{\star, 0}(R)+\Sigma_{\mathrm{DM}}(R),
$$

where $\Sigma_{\mathrm{DM}}(R)$ is the projected NFW profile. Disk galaxies generally have an exponential profile with Sérsic index $n \sim 1$.

The total density profiles in disk galaxies may be dominated either by dark matter or by luminous matter in their central regions. Even if dark matter dominates the spherically averaged density profile, in disk galaxies it provides a relatively smaller contribution to the torque if the cluster is embedded in the disk. This is because the torque coupling of the migrating star cluster with the flattened disk, Equation (5), is more significant than that with the dark matter halo (see, e.g., Bekki 2010). We use the disk torque to model the migration time in disk galaxies.

\subsection{Stellar Mass Redistribution by Cluster Migration}

We assume that all stars form in clusters with masses between $M_{\text {min }}$ and $M_{\max }$ (see Section 2.1). Let $d^{2} n / d M d r$ be the number of clusters per unit initial cluster mass per unit radius that have formed in a galaxy such that $\int_{0}^{\infty}\left(d^{2} n / d M d r\right) d r$ is proportional to the ICMF in Equation (1), while

$$
\int_{M_{\min }}^{M_{\max }} \frac{d^{2} n}{d M d r} M d M= \begin{cases}4 \pi r^{2} \rho_{\star, 0}(r), & \text { spheroid } \\ 2 \pi r \Sigma_{\star, 0}(r), & \text { disk. }\end{cases}
$$

We sample cluster masses and initial radii randomly according to the distribution $d^{2} n / d M d r$. The total number of clusters in our simulations is $\int_{M_{\min }}^{M_{\max }}(d n / d M) d M \approx$ $M_{\text {tot }} /\left[M_{\min } \ln \left(M_{\max } / M_{\min }\right)\right]$, where $M_{\text {tot }}$ is the total stellar mass, $M_{\text {sph }}$ or $M_{\text {disk }}$, of the galaxy. At first, we do not explicitly take into account the possible prompt, possibly mass-independent (for a discussion of mass-dependent prompt dissolution see, e.g., Goodwin 2009) dissolution of clusters ("infant mortality," see, e.g., Fall et al. 2005; Bastian \& Goodwin 2006; Chandar et al. 2006; Goodwin \& Bastian 2006; Gieles et al. 2007; Pellerin et al. 2007; Whitmore et al. 2007; Baumgardt et al. 2008; de Grijs \& Goodwin 2008; Lamers 2009). Then, we briefly assess the effects of prompt dissolution.

We proceed to model cluster migration and dissolution by following the cluster orbital decay

$$
\frac{d r}{d t}=-\frac{r}{t_{\mathrm{mig}}}
$$

and mass loss

$$
\frac{d M}{d t}=-\frac{M}{t_{\mathrm{dis}}},
$$

where $t_{\text {mig }}$ and $t_{\text {dis }}$ are, respectively, the migration timescale in Equation (3) and dissolution timescale in Equation (6).

We calculate the orbital decay of clusters in the order of increasing migration time (see Equation (3)) evaluated before migration has occurred. We keep track of the mass tidally stripped from the cluster with $k$ th shortest migration time by calculating its contribution to the stellar density profile

$$
\Delta \rho_{\star, k}=\frac{1}{4 \pi r_{k}^{2}} \frac{d M_{k}}{d t} / \frac{d r_{k}}{d t} \quad\left(r_{k}<r_{k, 0}\right),
$$

where $r_{k, 0}$ is the cluster formation radius and $k=1,2, \ldots, n_{\text {tot }}$. We assume that a cluster has been fully disrupted when its mass falls below $M_{\min }=100 M_{\odot}$ since in clusters of this size $t_{\mathrm{mig}} \gg t_{\mathrm{dis}}$ and thus the cluster is not able to migrate substantially, if at all, before disruption; this residual mass is deposited at the radius of disruption. Some migrating clusters avoid complete disruption until they reach the innermost radius 
of our grid, $r_{\min }=1 \mathrm{pc}$. The migrating cluster mass that reaches the central parsec is added to the central point mass $M_{\text {cent }}$. To account for the influence of the growing nuclear component, including $M_{\text {cent }}$ and the mass deposited at larger radii, e.g., at $r \lesssim 100 \mathrm{pc}$, on the migration and disruption timescales of more slowly inspiraling clusters, we calculate the tidally stripped mass of faster migrating star clusters with smaller $t_{\text {mig }}$ first. After the migration and disruption of $k$ clusters has been computed, we sum up their contributions to the modified stellar density profile of the galaxy $\Delta \rho_{\star}^{(k)}=\sum_{k^{\prime}=1}^{k} \Delta \rho_{\star, k^{\prime}}$ and set the stellar density profile in which the $(k+1)$ st cluster migrates to $\max \left(\rho_{\star, 0}, \Delta \rho_{\star}^{(k)}\right)$ so that the late-migrating clusters do so in a galaxy modified by the central concentration increase from the early-migrating clusters. We take the final density profile of the galaxy, after all clusters have been disrupted, to be $\rho_{\star}=\sum_{k} \Delta \rho_{\star, k}$. This prescription does not account for the escape of the material liberated by stellar mass loss from the galaxy. To model the effect of this escape, we repeat the calculation after requiring the first $50 \%$ of the mass to be stripped from a cluster leave the galaxy.

We treat the mass delivered to the central parsec as an "unresolved" stellar nucleus component with mass $M_{\text {cent. }}$ We model its surface density profile with the function

$$
\Sigma_{\text {cent }}(R)=\frac{M_{\text {cent }} R_{\mathrm{cent}}^{2}}{\pi\left(R_{\mathrm{cent}}^{2}+R^{2}\right)^{2}},
$$

where $R_{\text {cent }}$ is the half-light radius of the unresolved component. We set this radius to $R_{\text {cent }}=2 \mathrm{pc}$, which is chosen to be larger than our innermost grid radius but smaller than typical observed half-light radii of NSCs, e.g., $R_{\text {nuc }} \gtrsim 10$ pc (e.g., Geha et al. 2002). We add this unresolved component to the surface density profile calculated from the mass deposited by migrating clusters at $r>1 \mathrm{pc}$, so that

$$
\Sigma_{\star, \text { total }}(R)=\int_{-\infty}^{\infty} \rho_{\star}\left[\left(R^{2}+z^{2}\right)^{1 / 2}\right] d z+\Sigma_{\text {cent }}(R) .
$$

Our surface density profiles do not take into account any smearing by the point-spread function (PSF). If the PSF width is comparable to the resulting NSC radius, then smearing by the PSF must be taken into account when comparing synthesized surface brightness profiles with the observed ones. Because our results are affected by a variety of crude approximations we do not proceed to model the effect of the PSF.

\subsection{Limitations of the Model}

Before proceeding to discuss our results we would like to highlight the assumptions and limitations of our model. We have assumed that all clusters form simultaneously and thus we calculated the orbital decay of the clusters in order from fastest migrating (as calculated before any migration has occurred) to slowest migrating. This assumption is applicable to those dwarf galaxies that consume gas and form stars in a single star formation episode. In other galaxies, including late-type disks, however, cluster formation is ongoing (e.g., in galaxy M33) or is triggered by galactic mergers (e.g., in the Antennae galaxies). Our model could be adapted to approximate ongoing star formation by calculating cluster orbital decay and the associated mass transport in random order so that an older, less massive, and slower-migrating cluster can migrate and deposit its mass before a younger, more massive, and faster-migrating cluster does. Without migration-time ordering, our resulting NSC masses are similar to those in the corresponding ordered models. We do not attempt to estimate the timescale for the buildup of NSC mass in this scenario because the total timescale, although certainly longer than in the instantaneous star formation scenario, would depend on additional parameters - the star formation rate and the rate of gas accretion onto the galaxy - that are bound to vary between galaxies.

In an effort to focus on the variation in the galactic surface density profile and NSC mass buildup as a function of the ICMF truncation mass scale $M_{\max }$, we do not study their dependence on other parameters such as galaxy mass and initial concentration (Sérsic index) or on parameters, such as the coefficients characterizing the amplitudes of the dynamical friction torques driving cluster migration, that are subject to theoretical uncertainty. In the same spirit, we do not consider star formation episodes resulting from galactic mergers as this would require separate tracking of the galactic stellar and gas masses. The role of gaseous accretion and galactic mergers in galaxy assembly is best addressed with comprehensive cosmological hydrodynamical simulations (e.g., Governato et al. 2004, 2007, 2009; Kaufmann et al. 2007; Brooks et al. 2009; Ceverino et al. 2010), as analytical and semi-analytical models are inadequate in this context. We also do not model the response of the dark matter halo to the initial baryonic infall and to the subsequent evolution due to young cluster migration and stellar mass loss. Our model for progressive cluster migration and decay is crude and thus we will compare only the general characteristics of the resulting surface density profiles with those of observed galactic profiles. We caution against comparison with the detailed features of observed galaxy photometry.

\section{RESULTS}

We are ready to define the parameters of our sample calculations and provide results for model spheroidal and disk galaxies forming at high and low redshifts. For a fixed effective radius of the initial stellar density profile, the formation redshift determines the average density of dark matter within the effective radius. High-redshift models have dark matter halos with lower concentration and smaller virial radius than low-redshift models. The parameters $M_{\text {halo }}, c$, and $r_{\text {vir }}$, in general, depend on the assumed cosmological model and on the redshift of halo formation. We assume a flat universe with $\Omega_{\mathrm{m}}=0.274, \Omega_{\Lambda}=0.726$, and $h=0.705$ (Komatsu et al. 2009). The mass of the halo is related to the virial radius by $M_{\text {halo }}=\frac{4}{3} \pi \Delta_{\mathrm{c}}(z) \rho_{\text {crit }} r_{\text {vir }}^{3}$, where $\rho_{\text {crit }}$ and $\Delta_{\mathrm{c}}(z)$ are, respectively, the critical density of the universe and the mean overdensity within the virial radius at redshift $z$ (Bryan \& Norman 1998). For example, $\Delta_{\mathrm{c}}(0) \approx 100$ and $\Delta_{\mathrm{c}}(5-10) \approx 177$. The median concentration parameter for all high-redshift dark matter halos is $c \approx 3.5$, but at low redshift varies steeply with halo mass (e.g., Bullock et al. 2001; Zhao et al. 2003; Gao et al. 2008; Klypin et al. 2010). Our high- and low-redshift models are for $z=6$ and $z=0$, respectively.

We present our results for spheroidal and disk galaxies in Sections 4.1 and 4.2, respectively. Then in Section 4.3 we explore the observationally motivated scenario in which a large fraction of the clusters dissolve promptly following formation.

\subsection{Spheroidal Galaxies}

In Figure 1, left and middle panels, we show the projected surface density profiles for stars before migration and disruption $\left(M_{\mathrm{sph}}=10^{9} M_{\odot}, R_{\mathrm{s}}=0.5 \mathrm{kpc}\right.$, and $\left.n=1.5\right)$, for dark matter $\left(M_{\text {halo }}=10^{10} M_{\odot}\right.$, high- $z: c=3.5, r_{\text {vir }}=10 \mathrm{kpc}$; low- $z: c=$ 

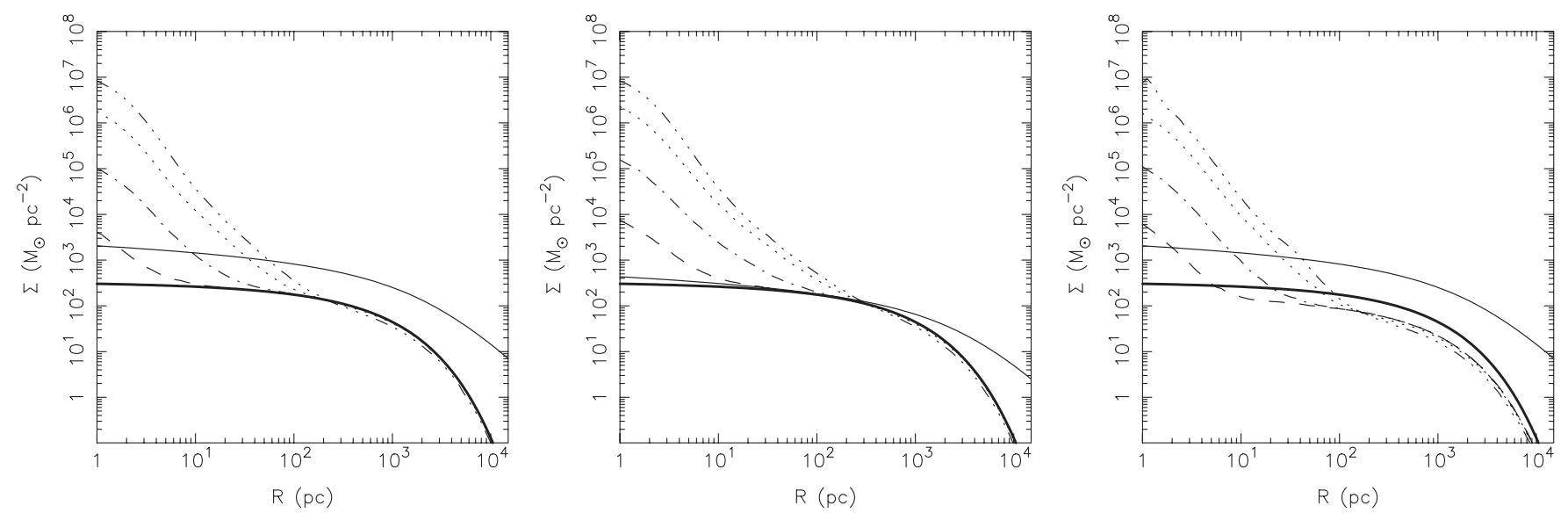

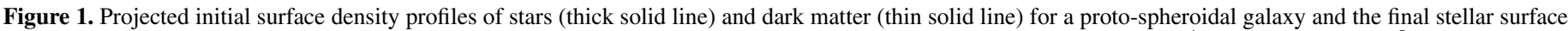

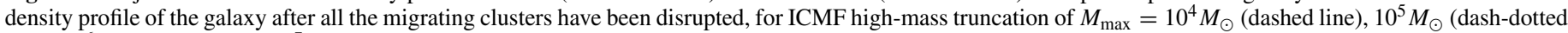

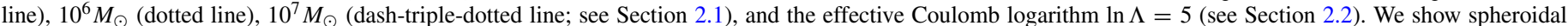

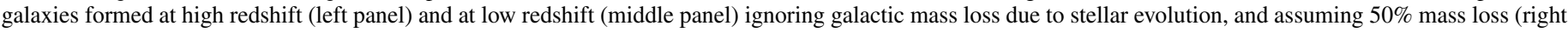
panel; see Section 3.3).

$15, r_{\mathrm{vir}}=60 \mathrm{kpc}$ ), and for stars after migration and disruption, assuming $\ln \Lambda=5$ and $M_{\max }=\left(10^{4}, 10^{5}, 10^{6}, 10^{7}\right) M_{\odot}$. To simplify our calculation, we take $p=2 / 3$ instead of the value $p \approx 0.62$ implied by Equation (10). In these models, we have not taken into account galactic mass loss resulting from stellar evolution. To explore the consequences of potential galactic loss of gas liberated by supernovae and asymptotic giant branch stars, in Figure 1, right panel, we repeat the calculation for the high-redshift model assuming that the first $50 \%$ of the mass dissociated from each migrating cluster is lost, i.e., leaves the galaxy. The models shown in Figure 1 do not allow for prompt cluster dissolution; we explore the impact of prompt dissolution in Section 4.3 below.

The final structure of the galaxy, after all clusters have migrated and dissolved, is a strong function of the ICMF truncation mass scale $M_{\max }$. For $M_{\max }=10^{4} M_{\odot}$, the excess surface density above the initial stellar density profile is present only in the central $\sim 10 \mathrm{pc}$ and is small. At the other end of the range of ICMF truncation mass scales, for $M_{\max }=10^{7} M_{\odot}$, the surface density profile of the entire galaxy has become slightly more concentrated, but still approximately follows the initial Sérsic profile at large radii. For $M_{\max }=\left(10^{4}-10^{7}\right) M_{\max }$, the resulting surface density excess after cluster migration and disruption appears as a distinct departure from the inwardextrapolated outer Sérsic law at $R \sim 20-200 \mathrm{pc}$ in the high$z$ model and $R \sim 30-300 \mathrm{pc}$ in the low- $z$ model; the excess surface density $\Sigma_{\star, \text { total }}-\Sigma_{\text {cent }}$ approximates power laws $\propto R^{-0.6}$ $R^{-1.7}$. There is little difference between the profiles calculated at low and high redshifts; this could in part be attributed to the cancellation of the nearly opposite effects of increasing halo concentration and decreasing mean halo density with decreasing redshift. Galactic stellar mass loss from stellar evolution leads to a decrease of stellar surface density at all radii but does not modify the overall character of the final surface density profile.

In Figure 2, left panel, we show the evolution of the NSC mass proxy $M_{10 \mathrm{pc}}$, here simply calculated as the stellar mass contained in a sphere with radius $r=10 \mathrm{pc}$ as a function of the initial migration time of the $k$ th cluster to migrate to the galaxy center (see Section 3.3). The latter is an approximation since the migration time evolves as the cluster loses mass and as its orbit decays, and as the galactic potential is modified by the previous $k-1$ migrating clusters. The initial value of $M_{10 \mathrm{pc}}$ in Figure 2,
Table 1

Spheroidal Galaxy ${ }^{\mathrm{a}}$ NSC Properties

\begin{tabular}{lccccc}
\hline \hline Model & $\begin{array}{c}\log \left(M_{\max }\right) \\
\left(M_{\odot}\right)\end{array}$ & $\begin{array}{c}\log \left(t_{1 / 2}\right) \\
(\mathrm{yr})\end{array}$ & $\begin{array}{c}\log \left(M_{10 \mathrm{pc}}\right) \\
\left(M_{\odot}\right)\end{array}$ & $\begin{array}{c}M_{10 \mathrm{pc}} / M_{\text {sph }} \\
(\mathrm{dex})\end{array}$ & $\begin{array}{c}M_{10 \mathrm{pc}} / M_{\max } \\
(\mathrm{dex})\end{array}$ \\
\hline High- $z$ & 4 & 7.96 & 4.87 & -4.13 & 0.87 \\
& 5 & 8.60 & 6.32 & -2.68 & 1.32 \\
& 6 & 9.50 & 7.52 & -1.48 & 1.52 \\
& 7 & 9.34 & 8.20 & -0.80 & 1.20 \\
\hline Low- $z$ & 4 & 8.09 & 5.24 & -3.76 & 1.24 \\
& 5 & 8.83 & 6.51 & -2.49 & 1.51 \\
& 6 & 9.59 & 7.64 & -1.36 & 1.64 \\
& 7 & 9.13 & 8.21 & -0.79 & 1.21 \\
\hline
\end{tabular}

Note. ${ }^{\text {a }} M_{\text {sph }}=10^{9} M_{\odot}, M_{\text {halo }}=10^{10} M_{\odot}$, no galactic mass loss, or prompt dissolution.

ranging from $\sim 10^{2} M_{\odot}$ to $>10^{6} M_{\odot}$, corresponds to the initial mass of the fastest-migrating cluster in each simulation; for $M_{\max }=10^{7} M_{\odot}$, the mass of the fastest-migrating cluster to reach $r<10 \mathrm{pc}$ already falls within the range of observed NSC masses (see Section 5.1). The mass $M_{10 \mathrm{pc}}$ contains $96 \%$ of the mass of the unresolved nucleus $M_{\text {cent }}$ accounting for the cluster stars that have reached $r=1 \mathrm{pc}$ and an additional "resolved" mass deposited by clusters migrating from $10 \mathrm{pc}$ to $1 \mathrm{pc}$. The mass $M_{10 \mathrm{pc}}$ increases as an approximate power law in time with slopes between $M_{10 \mathrm{pc}} \propto t^{1 / 2}$ and $M_{10 \mathrm{pc}} \propto t$ and then levels off. In Table 1, we report the final asymptotic $M_{10 \mathrm{pc}}$ and its ratio to the total stellar mass, as well as the total time $t_{1 / 2}$ that it takes for $M_{10 \mathrm{pc}}$ to reach one-half of its final value. In all the models presented in this work, the contribution of the unresolved central point mass $M_{\text {cent }}$ to $M_{10 \text { pc }}$ increases from about $40 \%$ for $M_{\max }=10^{4} M_{\odot}$ to $86 \%$ for $M_{\max }=10^{7} M_{\odot}$ in spheroidals and from about $20 \%$ for $M_{\max }=10^{4} M_{\odot}$ to $80 \%$ for $M_{\max }=10^{7} M_{\odot}$ in disks. We find that $>90 \%$ of the NSC mass is composed of clusters with initial masses $>0.1 M_{\max }$, indicating that the lowest-mass clusters make up only a small fraction of the NSC mass and, thus, that the final central mass is not sensitive to $M_{\min }$.

The asymptotic $M_{10 \mathrm{pc}}$ is a strong function of the ICMF truncation mass scale $M_{\max }$, as is the timescale for approach to this asymptotic mass. Larger $M_{\max }$ yield larger final central 

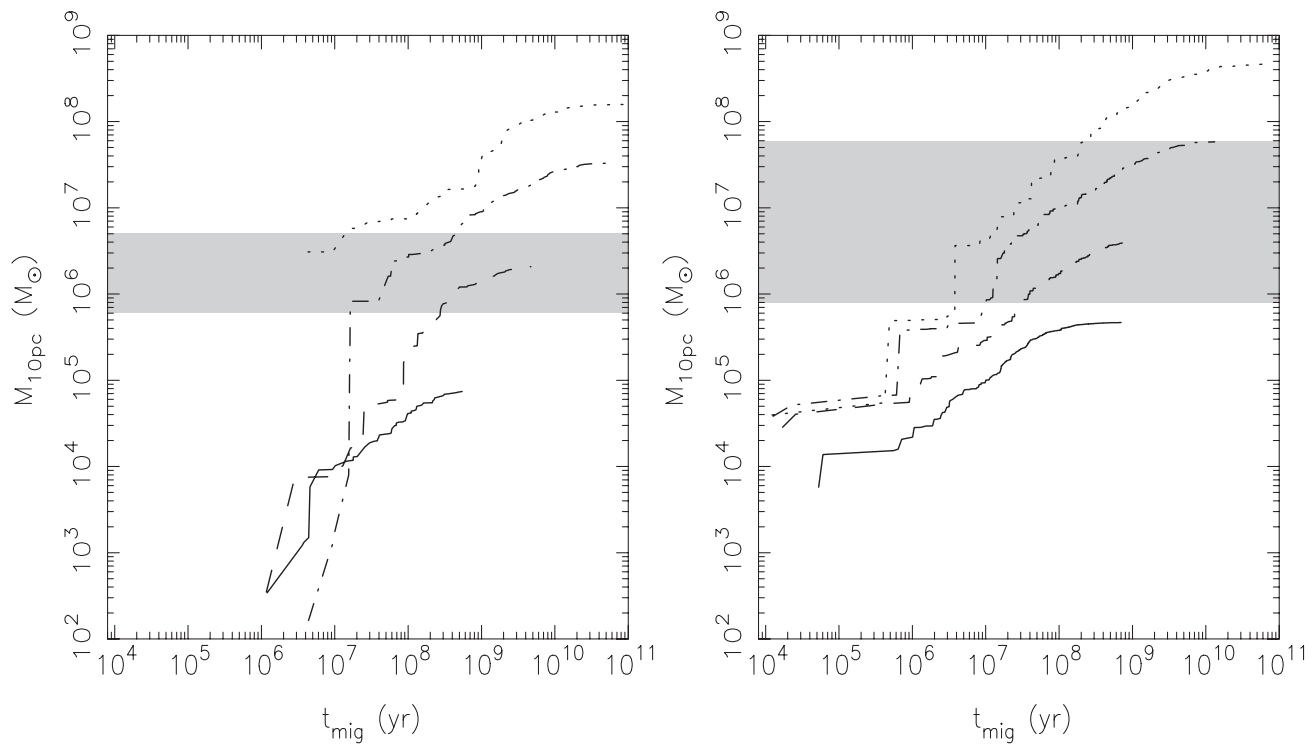

Figure 2. Total mass $M_{10 \mathrm{pc}}$ accumulated after migration and disruption of star clusters within the central $10 \mathrm{pc}$ of a high-redshift spheroidal galaxy (left panel) and a disk galaxy (right panel) as a function of migration time for those clusters that reach the central $10 \mathrm{pc}$ for $M_{\max }=10^{4} M_{\odot}$ (solid line), $M_{\max }=10^{5} M_{\odot}$ (dashed line), $10^{6} M_{\odot}$ (dash-dotted line), and $10^{7} M_{\odot}$ (dotted line). The mass histories end when the last cluster makes it to the central $10 \mathrm{pc}$. The shaded region in the left panel shows the $1 \sigma$ range of NSC mass-to-total stellar mass ratios from Ferrarese et al. (2006b) and Côté et al. (2006). In the right panel, the shaded region shows the range of NSC masses in Walcher et al. (2005).

masses that are assembled on longer timescales; this is because more massive clusters are able to migrate from larger radii. The central mass-to-ICMF truncation mass ratios are $M_{10 \mathrm{pc}} / M_{\max } \sim$ $1-1.5 \mathrm{dex}$, and thus the central stellar system is assembled from many migrating clusters. The scaling of the resulting NSC mass with the ICMF characteristic mass scale is consistent with the trend recognized by Bekki (2010), who carried out $N$-body integrations with an initial lognormal cluster mass function that approximates the evolved present-day globular cluster mass distribution.

\subsection{Disk Galaxies}

In the disk galaxy calculation, we choose $n=1$ and assume an approximate gas or stellar velocity dispersion in Equation (5) of $\sigma(r)=0.2\left[G M_{\mathrm{gal}}(r) / r\right]^{1 / 2}$, where $M_{\mathrm{gal}}(r)=$ $\max \left[M_{\star, 0}(r), M_{\star, k}(r)\right]+M_{\mathrm{DM}}(r)+M_{\text {cent }, k}(r)$ (see Section 3.3). Figure 3 in its left and middle panels shows the projected surface density profiles for stars $\left(M_{\text {disk }}=5 \times 10^{9} M_{\odot}, R_{\mathrm{S}}=1.5 \mathrm{kpc}\right.$, and $n=1)$, dark matter $\left(M_{\text {halo }}=5 \times 10^{10} M_{\odot}\right.$, high- $z: c=3.5$, $r_{\mathrm{vir}}=20 \mathrm{kpc}$; low- $\left.z: c=15, r_{\mathrm{vir}}=100 \mathrm{kpc}\right)$, and stars after migration and disruption for $M_{\max }=\left(10^{4}, 10^{5}, 10^{6}, 10^{7}\right) M_{\odot}$. As we did for spheroidal galaxies in Section 4.1, here we test the effect of galactic mass loss due to stellar evolution in Figure 3, right panel. The character of a disk galaxy's morphological transformation in consequence of cluster migration is very similar to that observed in spheroidal galaxies. The densities at smaller radii $\lesssim 10 \mathrm{pc}$ in disk galaxy models are somewhat higher than in spheroidal galaxy models. This may be explained by a higher initial central stellar surface density in our disk models. In Figure 2, right panel, we show the evolution of the mass enclosed within $r=10 \mathrm{pc}$ and in Table 2 we show the final, asymptotic mass $M_{10 \mathrm{pc}}$ and the timescale $t_{1 / 2}$ for accumulation of a half the final mass in disk galaxy models. We find that $M_{10 \mathrm{pc}} / M_{\max } \sim 1.7 \mathrm{dex}$ and that $>90 \%$ of the NSC mass is composed of clusters with initial masses above $0.1 M_{\max }$ for $M_{\max }>10^{4} M_{\odot}$ indicating that, again, the central stellar system is assembled from many migrating clusters and that the NSC mass is not sensitive to $M_{\min }$.
Table 2

Disk Galaxy ${ }^{\mathrm{a}}$ NSC Properties

\begin{tabular}{lccccc}
\hline \hline Model & $\begin{array}{c}\log \left(M_{\max }\right) \\
\left(M_{\odot}\right)\end{array}$ & $\begin{array}{c}\log \left(t_{1 / 2}\right) \\
(\mathrm{yr})\end{array}$ & $\begin{array}{c}\log \left(M_{10 \mathrm{pc}}\right) \\
\left(M_{\odot}\right)\end{array}$ & $\begin{array}{c}M_{10 \mathrm{pc}} / M_{\text {disk }} \\
(\mathrm{dex})\end{array}$ & $\begin{array}{c}M_{10 \mathrm{pc}} / M_{\max } \\
(\mathrm{dex})\end{array}$ \\
\hline High-z & 4 & 7.49 & 5.67 & -4.03 & 1.67 \\
& 5 & 8.08 & 6.60 & -3.10 & 1.60 \\
& 6 & 8.87 & 7.77 & -1.93 & 1.77 \\
& 7 & 9.29 & 8.67 & -1.03 & 1.67 \\
\hline Low- $z$ & 4 & 7.44 & 5.75 & -3.95 & 1.75 \\
& 5 & 8.01 & 6.68 & -3.02 & 1.68 \\
& 6 & 8.92 & 7.86 & -1.84 & 1.86 \\
& 7 & 9.10 & 8.69 & -1.00 & 1.69 \\
\hline
\end{tabular}

Note. ${ }^{\text {a }} M_{\text {disk }}=5 \times 10^{9} M_{\odot}, M_{\text {halo }}=5 \times 10^{10} M_{\odot}$, no galactic mass loss, or prompt dissolution.

\subsection{Prompt Cluster Dissolution}

Observations of star cluster populations in nearby disk galaxies suggest that a large $(\sim 90 \%)$, though still highly uncertain, fraction of young clusters dissolve very quickly after formation, likely as the result of an initial loosely bound state and rapid expulsion of the natal gas cloud from the cluster by photoevaporation and radiation pressure (this is often referred to as "infant mortality," see references cited in Section 3.3 above). There is evidence that the likelihood of prompt dissolution is independent of cluster mass. To explore the impact of prompt dissolution on the morphological transformation of the galaxy, we repeated the calculations carried out in Sections 4.1 and 4.2, but assuming that $90 \%$ of randomly selected clusters dissolve instantaneously and deposit their entire mass at the radius at which they formed. If prompt dissolution is mass dependent with, for example, significantly less than $90 \%$ of high-mass clusters being instantaneously dissolved, then we expect the resulting NSC mass from our simulations to be larger. The resulting stellar surface density profiles are shown in Figure 4. Now, even for our largest ICMF truncation mass scale of $M_{\max }=10^{7} M_{\odot}$, visible evolution of 

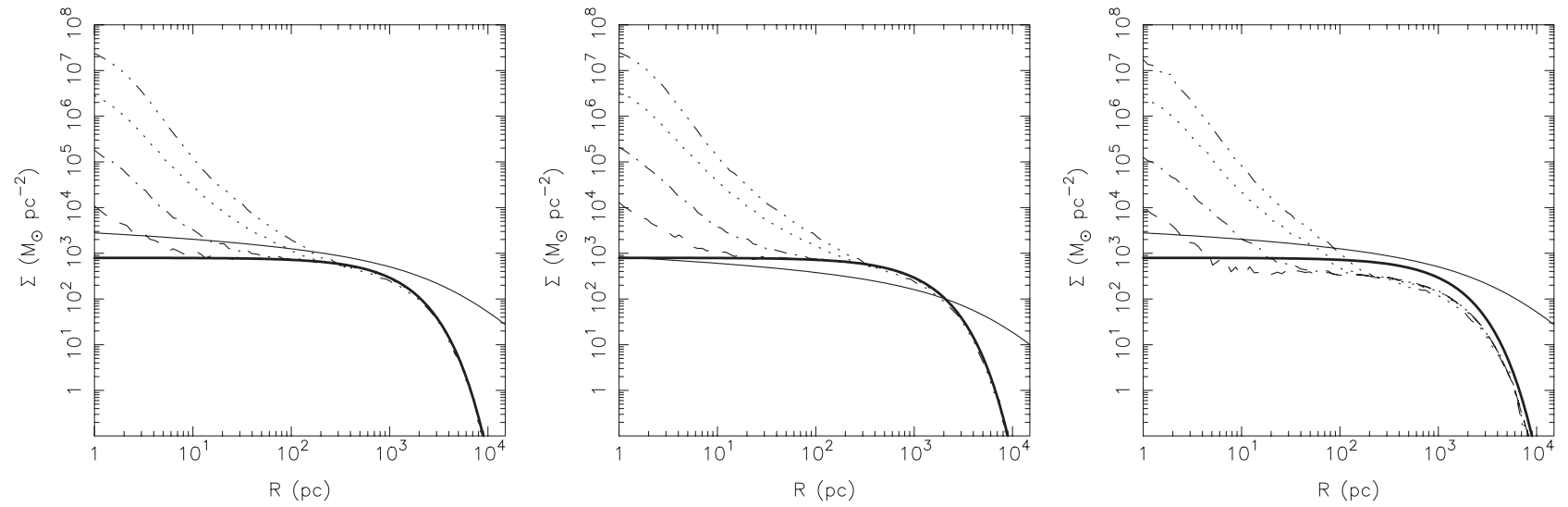

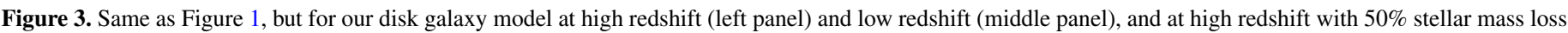
(right panel).
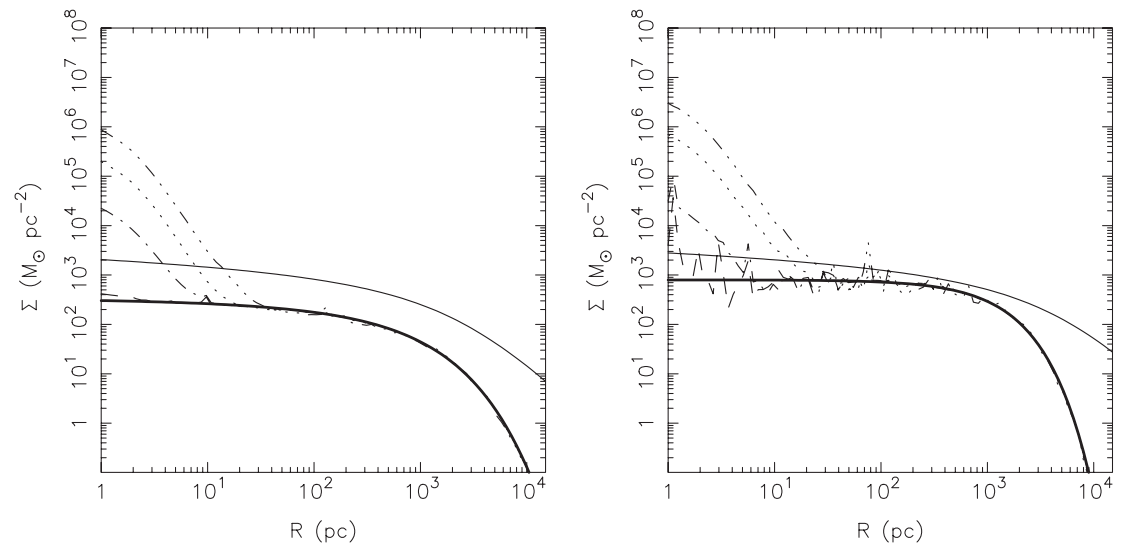

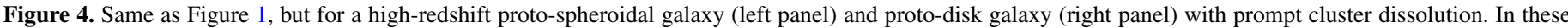
models, $90 \%$ of the clusters dissolve immediately independent of their mass, and the remaining $10 \%$ migrate inward prior to disruption.
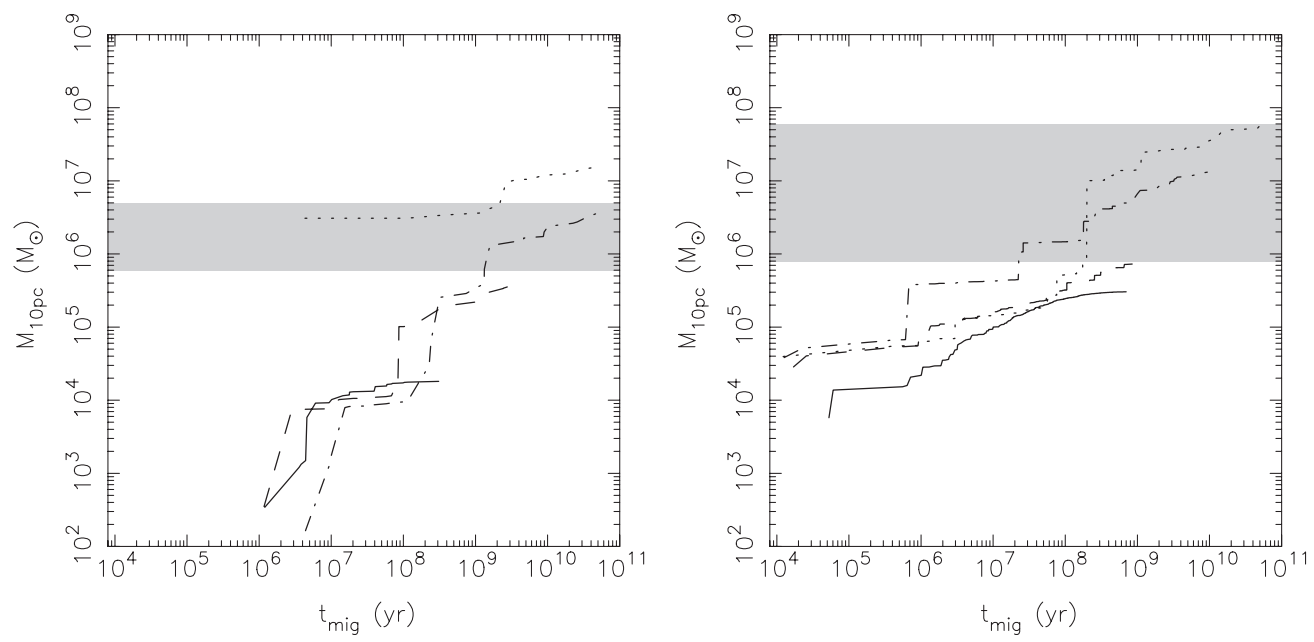

Figure 5. Same as Figure 2, except that $90 \%$ of the clusters are dissolved immediately independent of their mass (see Section 4.3).

the stellar surface density profile is confined within the inner $\sim 10-50 \mathrm{pc}$. The time evolution of the mass contained in the innermost $r=10 \mathrm{pc}$ is shown in Figure 5. The central masses rise as $M_{10 \mathrm{pc}} \propto t^{2 / 5}$ in most cases and reach smaller asymptotic values than without prompt dissolution. For $M_{\max }=10^{4} M_{\odot}$, there is no substantial departure from the inward-extrapolated Sérsic profile. For $M_{\max }=\left(10^{5}-10^{7}\right) M_{\odot}$, the central masses decrease from $\sim(10 \%$ to $20 \%)$ of the values calculated without prompt dissolution in spherical and disk galaxies. In Tables 3 and 4, we summarize the properties of the NSCs (see Section 3.1) in the simulations with $90 \%$ prompt cluster disso- lution and find that $M_{10 \mathrm{pc}} / M_{\max } \sim 0.2-0.8 \mathrm{dex}$ in spheroidals and $M_{10 \mathrm{pc}} / M_{\max } \sim 0.8-0.5 \mathrm{dex}$ in disks. The timescale $t_{1 / 2}$ for half of the final NSC mass to accumulate extends up to $10 \mathrm{Gyr}$.

\section{DISCUSSION}

\subsection{Comparison with Observed NSCs and Pseudobulges}

Our model calculations show that cluster migration can bring about galactic morphological transformation in which a new stellar density component grows at the center of the galaxy. The new component is a drastic departure from the 
Table 3

Spheroidal Galaxy ${ }^{\mathrm{a}}$ NSC Properties-90\% Prompt Dissolution

\begin{tabular}{cccccc}
\hline \hline Model & $\begin{array}{c}\log \left(M_{\max }\right) \\
\left(M_{\odot}\right)\end{array}$ & $\begin{array}{c}\log \left(t_{1 / 2}\right) \\
(\mathrm{yr})\end{array}$ & $\begin{array}{c}\log \left(M_{10 \mathrm{pc}}\right) \\
\left(M_{\odot}\right)\end{array}$ & $\begin{array}{c}M_{10 \mathrm{pc}} / M_{\mathrm{sph}} \\
(\mathrm{dex})\end{array}$ & $\begin{array}{c}M_{10 \mathrm{pc}} / M_{\max } \\
(\mathrm{dex})\end{array}$ \\
\hline High- $z$ & 4 & 6.66 & 4.26 & -4.74 & 0.26 \\
& 5 & 8.56 & 5.60 & -3.40 & 0.60 \\
& 6 & 9.94 & 6.55 & -2.45 & 0.55 \\
& 7 & 9.33 & 7.19 & -1.81 & 0.19 \\
\hline Low- $z$ & 4 & 8.23 & 4.70 & -4.30 & 0.70 \\
& 5 & 9.14 & 5.87 & -3.13 & 0.87 \\
& 6 & 10.00 & 6.75 & -2.25 & 0.75 \\
& 7 & 9.63 & 7.24 & -1.76 & 0.24 \\
\hline
\end{tabular}

Note. ${ }^{\mathrm{a}} M_{\mathrm{sph}}=10^{9} M_{\odot}, M_{\text {halo }}=10^{10} M_{\odot}$, and no galactic mass loss.

Table 4

Disk Galaxy ${ }^{\mathrm{a}}$ NSC Properties_-90\% Prompt Dissolution

\begin{tabular}{lccccc}
\hline \hline Model & $\begin{array}{c}\log \left(M_{\max }\right) \\
\left(M_{\odot}\right)\end{array}$ & $\begin{array}{c}\log \left(t_{1 / 2}\right) \\
(\mathrm{yr})\end{array}$ & $\begin{array}{c}\log \left(M_{10 \mathrm{pc}}\right) \\
\left(M_{\odot}\right)\end{array}$ & $\begin{array}{c}M_{10 \mathrm{pc}} / M_{\mathrm{disk}} \\
(\mathrm{dex})\end{array}$ & $\begin{array}{c}M_{10 \mathrm{pc}} / M_{\max } \\
(\mathrm{dex})\end{array}$ \\
\hline High- $z$ & 4 & 7.42 & 5.48 & -4.22 & 1.48 \\
& 5 & 8.20 & 5.92 & -3.78 & 0.92 \\
& 6 & 8.96 & 7.12 & -2.58 & 1.12 \\
& 7 & 9.75 & 7.75 & -1.95 & 0.75 \\
\hline \multirow{2}{*}{ Low- $z$} & 4 & 7.31 & 5.51 & -4.19 & 1.51 \\
& 5 & 8.25 & 6.05 & -3.65 & 1.05 \\
& 6 & 9.39 & 7.23 & -2.47 & 1.23 \\
& 7 & 9.36 & 7.85 & -1.85 & 0.85 \\
\hline
\end{tabular}

Note. ${ }^{\mathrm{a}} M_{\text {disk }}=5 \times 10^{9} M_{\odot}, M_{\text {halo }}=5 \times 10^{10} M_{\odot}$, and no galactic mass loss.

inward-extrapolated outer surface density profile of the galaxy, and this calls for comparison with NSCs in spheroidals and late-type disks and with central light excesses in spheroidals and pseudobulges in disks; we refer to both of the latter phenomena as "pseudobulges." The surface density profiles shown in Figures 1, 3, and 4 do not suggest a clear separation into a compact component that would be compared with an NSC and a more extended component that would be compared to a pseudobulge. We caution against overinterpreting the detailed profile because our model for progressive cluster mass loss and dissolution is crude and inevitably fails to accurately account for the evolution of the internal structure of the cluster. For example, if the cluster is dense enough for its core to collapse through mass segregation and two-body relaxation, then the cluster core could maintain integrity longer and would migrate to smaller radii than in our calculations. More accurate estimates of the detailed innermost stellar density profile can only be achieved with $N$-body simulations resolving the internal structure of the migrating clusters (see, e.g., Capuzzo-Dolcetta \& Miocchi 2008a, 2008b). With this caveat in mind, we pursue comparison of only the more robust characteristics of the calculated profiles with those of observed galaxies.

In Figures 2 and 4, which show the evolution of the mass $M_{10 \mathrm{pc}}$ contained in the innermost $10 \mathrm{pc}$, we indicate the range of NSC masses corresponding to the fractions of the spheroidal luminosity contained in the NSCs in the Virgo Cluster Survey of Ferrarese et al. (2006b) and Côté et al. (2006) and also indicate the range of absolute NSC masses in the survey of late-type disks by Böker et al. (2004) and Walcher et al. (2005). If $M_{10 \mathrm{pc}}$ is indeed a valid proxy for NSC mass, then this allows us to identify the range of ICMF truncation mass scales $M_{\max }$ consistent with the observed NSCs. The models not allowing for prompt dissolution are consistent with $M_{\max } \sim 10^{4}-10^{5} M_{\odot}$ for spheroidals and $M_{\max } \sim 10^{4}-10^{6} M_{\odot}$ for disks. The models with $90 \%$ prompt dissolution are consistent with $M_{\max } \sim$ $10^{5}-10^{6} M_{\odot}$ for spheroidals and $M_{\max } \sim 10^{5}-10^{7} M_{\odot}$ for disks. These estimates are consistent with the observationally inferred and theoretically anticipated values of the ICMF truncation mass scale (Section 2.1). The results uniformly exclude the possibility that the ICMF truncation mass scale in spheroidals and late-type disks is above $10^{7} M_{\odot}$.

The surface density profile modified by cluster migration already starts departing upward from the inward-extrapolated outer Sérsic (or exponential) law at a radius that increases with $M_{\max }=\left(10^{4}-10^{6}\right) M_{\odot}$ from $\sim 20 \mathrm{pc}$ to $\sim 300 \mathrm{pc}$ in the models without galactic mass loss and prompt dissolution. With galactic mass loss, the departure radius ranges from $\lesssim 10 \mathrm{pc}$ to $\sim 200 \mathrm{pc}$ for the same range of $M_{\max }$. These departure radii are relatively small fractions of the disk exponential scale length $R_{\mathrm{s}}$ in disk galaxies. In the models with $90 \%$ prompt dissolution, the departure radius ranges from $\sim 10 \mathrm{pc}$ to $\sim 40 \mathrm{pc}$ for $M_{\max }=\left(10^{5}-10^{7}\right) M_{\odot}$. For $M_{\max }=10^{4} M_{\odot}$, no significant surface density excess is present. Because of the crudeness of the prescriptions that we employ to model cluster migration and dissolution, and the sensitivity of the innermost density profile to these prescriptions, we do not attempt to fit an analytic profile, such as a two-component Sérsic profile allowing for a photometrically distinct central light excess (and perhaps a third component - the NSC), to the surface density profile of the final galaxy. Nevertheless, the excess we observe in the central one or few hundred parsecs is suggestive of pseudobulges that have been identified photometrically in disk galaxies (Kormendy \& Kennicutt 2004; Fisher \& Drory 2008, 2010; Fisher et al. 2009; Weinzirl et al. 2009) and the "central light excesses" identified in bulgeless disks (Böker et al. 2003), including the late-type disk M33 (Kent 1987; Minniti et al. 1993).

The effective radii of the pseudobulge components identified in the recent surveys by Fisher et al. and Weinzirl et al. seem to be compatible with our more optimistic models that ignore prompt dissolution and are on average larger than the radii within which we detect surface density excess in the pessimistic models with $90 \%$ prompt dissolution. We caution against direct comparison because in the present work, in an attempt to emphasize sensitivity to the variation of the ICMF truncation mass scale $M_{\max }$, we have held the parameters of our dark halo and initial baryonic disk (or spheroid) fixed at values that seem to correspond to galaxies that are somewhat smaller than the typical pseudobulge hosts. We can only conclude that a pseudobulge-like central stellar surface density increase is generic and that cluster migration is one potential contributor to pseudobulge assembly in disk galaxies, while other processes, such as angular momentum transport by stellar and gaseous bars, certainly also contribute in line with the observation that pseudobulge hosts generally have nuclear bars, rings, or nuclear spirals (e.g., Kormendy \& Kennicutt 2004; Fisher \& Drory 2008).

The most massive clusters that we have considered are still substantially less massive than the giant $\sim 10^{8}-10^{9} M_{\odot}$ clumps that are observed to be present and are theoretically expected to be forming in globally gravitationally unstable, rapidly starforming massive disks at high redshift (e.g., Noguchi 1999; Bournaud et al. 2007; Elmegreen et al. 2008b; Dekel et al. 2009a; Tacconi et al. 2010, and references therein). The super star clusters forming in these giant clumps should be more immune to dissolution in the tidal field of the galaxy and could 
reach the galactic central region intact. We speculate that there could be a critical characteristic ICMF mass scale above which clusters migrate intact and merge to produce a classical bulge (see, e.g., Immeli et al. 2004; Elmegreen et al. 2008b; Ceverino et al. 2010), and below which they suffer substantial mass loss en route to the galactic center and thus give rise to a pseudobulge.

\subsection{Implications for Massive Black Holes}

The apparent agreement of NSC-mass-to-galactic-stellarmass ratios in spheroidals $\left(\sim 2 \times 10^{-3}\right.$; Côté et al. 2006; Ferrarese et al. 2006b; Wehner \& Harris 2006) and massiveblack-hole-to-galactic stellar mass ratios in ellipticals and bulges ( $\sim(1-2) \times 10^{-3}$; e.g., Kormendy \& Richstone 1995; Wandel 1999; Kormendy \& Gebhardt 2001; Merritt \& Ferrarese 2001; McLure \& Dunlop 2002; Marconi \& Hunt 2003; Häring \& Rix 2004) has prompted speculation that the same process may be responsible for the formation of NSCs and black holes. While the formation and growth of a massive black hole undoubtedly require a gas-dynamical, dissipative process, our results suggest that an NSC can be assembled non-dissipatively by the collisionless migration of star clusters, and thus, the observed agreement could be a coincidence.

Although the well-studied NSC host galaxy M33 does not contain a central massive black hole (Merritt et al. 2001; Gebhardt et al. 2001, and references therein), another one, NGC 4395, does (Filippenko \& Ho 2003), and still others contain active galactic nuclei (AGNs; Seth et al. 2008). Since AGNs and the growth of a central black hole require gas inflow into the center of the galaxy, NSC growth from migrating disk clusters would not generally be accompanied with black hole growth, because disk clusters contribute stellar mass without augmenting the black hole mass (although gas inflow may be enhanced by the migrating clusters, see, e.g., Goodman \& Rafikov 2001; Chang 2008). This suggests that the central black hole mass in bulgeless disks should not be correlated with the mass of the NSC and any pseudobulge that is present, unless the migrating clusters independently synthesize massive (or intermediate-mass) black holes which, in this case, they would deliver to the center to merge to form a more massive central black hole (Elmegreen et al. 2008a).

\section{CONCLUSIONS}

We have studied the morphological transformation of isolated spheroidal and late-type disk galaxies driven by migration and tidal disruption of embedded star clusters. With the help of empirically and theoretically calibrated cluster migration and disruption timescales, we have tracked the mass that accumulates in the central region of a spheroidal or latetype disk galaxy and have compared the resulting innermost surface density profile to that of NSC and pseudobulge host galaxies. We have focused on the variation in the degree of galaxy transformation with the ICMF truncation mass scale; the assumptions and limitations of our model are described in Section 3.4. Our main conclusions are as follows.

1. The amount of stellar mass that the migrating clusters transport into the central few tens or hundreds of parsecs is sensitive to the maximum mass of the clusters forming in the galaxy. Larger ICMF high-mass truncation mass, $M_{\max }$, scales yield larger and more spatially extended central stellar accumulations.

2. Because our model for progressive cluster disruption is crude, we cannot attach significance to the detailed shape of the innermost surface density profile (the number of photometrically distinct components, inflection points, etc.), but we do compare the robust features of our synthetic profiles with the observed properties of NSC host galaxies.

3. Our model yields NSC masses compatible with the observed NSC masses and scaling relations if the ICMF truncation mass scale is $10^{5} M_{\odot} \lesssim M_{\max } \lesssim 10^{6} M_{\odot}$, which is consistent with direct $M_{\max }$ estimates in the nearby starforming galaxies. To match NSC masses in galaxies with a higher degree of prompt dissolution ("infant mortality"), higher ICMF truncation mass scales are needed.

4. The surface density profiles modified by cluster migration exhibit excesses above the inward-extrapolated outer Sérsic (or exponential) profile in the central few tens to few hundreds of parsecs. These excesses are suggestive of the pseudobulge phenomenon in disk galaxies. The formation of NSCs and central surface density excesses in bulgeless galaxies is inevitable and generic simply as a consequence of the clustered nature of star formation.

We thank D. Fisher, M. Gieles, J. Kormendy, and C. J. Walcher for invaluable discussions and also thank the organizers of the workshop Nuclear Star Clusters across the Hubble Sequence at MPIA, Heidelberg, 2008. We also thank an anonymous referee for helpful comments on an earlier version of this work. We acknowledge NSF grant AST-0708795.

\section{REFERENCES}

Balcells, M., Graham, A. W., Domínguez-Palmero, L., \& Peletier, R. F. 2003, ApJ, 582, L79

Baruteau, C., \& Masset, F. 2008, ApJ, 678, 483

Bastian, N., \& Goodwin, S. P. 2006, MNRAS, 369, L9

Baumgardt, H., Kroupa, P., \& Parmentier, G. 2008, MNRAS, 384, 1231

Baumgardt, H., \& Makino, J. 2003, MNRAS, 340, 227

Bekki, K. 2010, MNRAS, 401, 2753

Bellazzini, M., et al. 2008, AJ, 136, 1147

Bik, A., Lamers, H. J. G. L. M., Bastian, N., Panagia, N., \& Romaniello, M. 2003, A\&A, 397, 473

Böker, T., Laine, S., van der Marel, R. P., Sarzi, M., Rix, H.-W., Ho, L. C., \& Shields, J. C. 2002, AJ, 123, 1389

Böker, T., Sarzi, M., McLaughlin, D. E., van der Marel, R. P., Rix, H.-W., Ho, L. C., \& Shields, J. C. 2004, AJ, 127, 105

Böker, T., Stanek, R., \& van der Marel, R. P. 2003, AJ, 125, 1073

Bournaud, F., Elmegreen, B. G., \& Elmegreen, D. M. 2007, ApJ, 670, 237

Boutloukos, S. G., \& Lamers, H. J. G. L. M. 2003, MNRAS, 338, 717

Brooks, A. M., Governato, F., Quinn, T., Brook, C. B., \& Wadsley, J. 2009, ApJ, 694, 396

Bryan, G. L., \& Norman, M. L. 1998, ApJ, 495, 80

Bullock, J. S., Kolatt, T. S., Sigad, Y., Somerville, R. S., Kravtsov, A. V., Klypin, A. A., Primack, J. R., \& Dekel, A. 2001, MNRAS, 321, 559

Caon, N., Capaccioli, M., \& D’Onofrio, M. 1993, MNRAS, 265, 1013

Capuzzo-Dolcetta, R., \& Miocchi, P. 2008a, MNRAS, 388, L69

Capuzzo-Dolcetta, R., \& Miocchi, P. 2008b, ApJ, 681, 1136

Ceverino, D., Dekel, A., \& Bournaud, F. 2010, MNRAS, 404, 2151

Chandar, R., Fall, S. M., \& Whitmore, B. C. 2006, ApJ, 650, L111

Chandar, R., et al. 2010, ApJ, 719, 966

Chang, P. 2008, ApJ, 684, 236

Côté, P., et al. 2006, ApJS, 165, 57

D’Angelo, G., Kley, W., \& Henning, T. 2003, ApJ, 586, 540

de Grijs, R., \& Anders, P. 2006, MNRAS, 366, 295

de Grijs, R., Anders, P., Bastian, N., Lynds, R., Lamers, H. J. G. L. M., \& O’Neil, E. J. 2003, MNRAS, 343, 1285

de Grijs, R., \& Goodwin, S. P. 2008, MNRAS, 383, 1000

Dekel, A., Sari, R., \& Ceverino, D. 2009a, ApJ, 703, 785

Dekel, A., et al. 2009b, Nature, 457, 451

Donner, K. J., \& Sundelius, B. 1993, MNRAS, 265, 88

Dowell, J. D., Buckalew, B. A., \& Tan, J. C. 2008, AJ, 135, 823

Dutton, A. A. 2009, MNRAS, 396, 121

Elmegreen, B. G., Bournaud, F., \& Elmegreen, D. M. 2008a, ApJ, 684, 829

Elmegreen, B. G., Bournaud, F., \& Elmegreen, D. M. 2008b, ApJ, 688, 67 
Fall, S. M., Chandar, R., \& Whitmore, B. C. 2005, ApJ, 631, L133

Fall, S. M., \& Zhang, Q. 2001, ApJ, 561, 751

Ferrarese, L., et al. 2006a, ApJS, 164, 334

Ferrarese, L., et al. 2006b, ApJ, 644, L21

Filippenko, A. V., \& Ho, L. C. 2003, ApJ, 588, L13

Fisher, D. B., \& Drory, N. 2008, AJ, 136, 773

Fisher, D. B., \& Drory, N. 2010, ApJ, 716, 942

Fisher, D. B., Drory, N., \& Fabricius, M. H. 2009, ApJ, 697, 630

Gao, L., Navarro, J. F., Cole, S., Frenk, C. S., White, S. D. M., Springel, V., Jenkins, A., \& Neto, A. F. 2008, MNRAS, 387, 536

Gebhardt, K., et al. 2001, AJ, 122, 2469

Geha, M., Guhathakurta, P., \& van der Marel, R. P. 2002, AJ, 124, 3073

Geha, M., Guhathakurta, P., \& van der Marel, R. P. 2003, AJ, 126, 1794

Genzel, R., et al. 2008, ApJ, 687, 59

Georgiev, I. Y., Hilker, M., Puzia, T. H., Goudfrooij, P., \& Baumgardt, H. 2009, MNRAS, 396, 1075

Gieles, M. 2009, MNRAS, 394, 2113

Gieles, M., \& Baumgardt, H. 2008, MNRAS, 389, L28

Gieles, M., Lamers, H. J. G. L. M., \& Baumgardt, H. 2008, in IAU Symp. 246, Dynamical Evolution of Dense Stellar Systems, ed. E. Vesperini, M. Giersz, \& A. Sills (Cambridge: Cambridge Univ. Press), 171

Gieles, M., Lamers, H. J. G. L. M., \& Portegies Zwart, S. F. 2007, ApJ, 668, 268

Gieles, M., Larsen, S. S., Bastian, N., \& Stein, I. T. 2006a, A\&A, 450, 129

Gieles, M., Larsen, S. S., Scheepmaker, R. A., Bastian, N., Haas, M. R., \& Lamers, H. J. G. L. M. 2006b, A\&A, 446, L9

Gieles, M., Portegies Zwart, S. F., Baumgardt, H., Athanassoula, E., Lamers, H. J. G. L. M., Sipior, M., \& Leenaarts, J. 2006c, MNRAS, 371, 793

Goldreich, P., \& Tremaine, S. 1980, ApJ, 241, 425

Goodman, J., \& Rafikov, R. R. 2001, ApJ, 552, 793

Goodwin, S. P. 2009, Ap\&SS, 324, 259

Goodwin, S. P., \& Bastian, N. 2006, MNRAS, 373, 752

Governato, F., Willman, B., Mayer, L., Brooks, A., Stinson, G., Valenzuela, O. Wadsley, J., \& Quinn, T. 2007, MNRAS, 374, 1479

Governato, F., et al. 2004, ApJ, 607, 688

Governato, F., et al. 2009, MNRAS, 398, 312

Graham, A. W., \& Guzmán, R. 2003, AJ, 125, 2936

Häring, N., \& Rix, H.-W. 2004, ApJ, 604, L89

Hopkins, P. F., Cox, T. J., Dutta, S. N., Hernquist, L., Kormendy, J., \& Lauer, T. R. 2009a, ApJS, 181, 135

Hopkins, P. F., Hernquist, L., Cox, T. J., Dutta, S. N., \& Rothberg, B. 2008, ApJ, 679,156

Hopkins, P. F., Lauer, T. R., Cox, T. J., Hernquist, L., \& Kormendy, J. 2009b, ApJS, 181, 486

Hunter, D. A., Elmegreen, B. G., Dupuy, T. J., \& Mortonson, M. 2003, AJ, 126, 1836

Immeli, A., Samland, M., Gerhard, O., \& Westera, P. 2004, A\&A, 413, 547

Kaufmann, T., Mayer, L., Wadsley, J., Stadel, J., \& Moore, B. 2007, MNRAS, 375,53

Kent, S. M. 1987, AJ, 94, 306

Kim, W.-T., Ostriker, E. C., \& Stone, J. M. 2003, ApJ, 599, 1157

Klypin, A., Trujillo-Gomez, S., \& Primack, J. 2010, arXiv:1002.3660

Koda, J., Milosavljević, M., \& Shapiro, P. R. 2009, ApJ, 696, 254

Komatsu, E., et al. 2009, ApJS, 180, 330

Kormendy, J., Drory, N., Bender, R., \& Cornell, M. E. 2010, ApJ, 723, 54

Kormendy, J., \& Fisher, D. B. 2008, in ASP Conf. Ser. 396, Formation and Evolution of Galaxy Disks, ed. J. G. Funes \& E. M. Corsini (San Francisco, CA: ASP), 297

Kormendy, J., Fisher, D. B., Cornell, M. E., \& Bender, R. 2009, ApJS, 182, 216

Kormendy, J., \& Gebhardt, K. 2001, in AIP Conf. 586, 20th Texas Symposium on Relativistic Astrophysics, ed. J. C. Wheeler \& H. Martel (Melville, NY: AIP), 363
Kormendy, J., \& Kennicutt, R. C., Jr. 2004, ARA\&A, 42, 603

Kormendy, J., \& Richstone, D. 1995, ARA\&A, 33, 581

Lamers, H. J. G. L. M. 2009, Ap\&SS, 324, 183

Lamers, H. J. G. L. M., Gieles, M., Bastian, N., Baumgardt, H., Kharchenko, N. V., \& Portegies Zwart, S. 2005a, A\&A, 441, 117

Lamers, H. J. G. L. M., Gieles, M., \& Portegies Zwart, S. F. 2005b, A\&A, 429, 173

Larsen, S. S. 2009, A\&A, 494, 539

Lima Neto, G. B., Gerbal, D., \& Márquez, I. 1999, MNRAS, 309, 481

Lotz, J. M., Telford, R., Ferguson, H. C., Miller, B. W., Stiavelli, M., \& Mack, J. 2001, ApJ, 552, 572

Marconi, A., \& Hunt, L. K. 2003, ApJ, 589, L21

McLure, R. J., \& Dunlop, J. S. 2002, MNRAS, 331, 795

McMillan, S. L. W., \& Portegies Zwart, S. F. 2003, ApJ, 596, 314

Merritt, D., \& Ferrarese, L. 2001, MNRAS, 320, L30

Merritt, D., Ferrarese, L., \& Joseph, C. L. 2001, Science, 293, 1116

Miller, B. W., \& Lotz, J. M. 2007, ApJ, 670, 1074

Milosavljević, M. 2004, ApJ, 605, L13

Minniti, D., Olszewski, E. W., \& Rieke, M. 1993, ApJ, 410, L79

Navarro, J. F., Frenk, C. S., \& White, S. D. M. 1997, ApJ, 490, 493

Noguchi, M. 1999, ApJ, 514, 77

Pellerin, A., Meyer, M., Harris, J., \& Calzetti, D. 2007, ApJ, 658, L87

Peñarrubia, J., Just, A., \& Kroupa, P. 2004, MNRAS, 349, 747

Peñarrubia, J., Kroupa, P., \& Boily, C. M. 2002, MNRAS, 333, 779

Portegies Zwart, S. F., Hut, P., Makino, J., \& McMillan, S. L. W. 1998, A\&A, 337,363

Portegies Zwart, S. F., Makino, J., McMillan, S. L. W., \& Hut, P. 2002, ApJ, 565,265

Prugniel, P., \& Simien, F. 1997, A\&A, 321, 111

Quinn, P. J., \& Goodman, J. 1986, ApJ, 309, 472

Rafelski, M., \& Zaritsky, D. 2005, AJ, 129, 2701

Rossa, J., van der Marel, R. P., Böker, T., Gerssen, J., Ho, L. C., Rix, H.-W., Shields, J. C., \& Walcher, C.-J. 2006, AJ, 132, 1074

Schaye, J. 2004, ApJ, 609, 667

Schinnerer, E., Böker, T., Emsellem, E., \& Downes, D. 2007, A\&A, 462, L27

Sérsic, J. L. 1963, Boletin de la Asociacion Argentina de Astronomia La Plata Argentina, 6, 41

Seth, A., Agueros, M., Lee, D., \& Basu-Zych, A. 2008, ApJ, 678, 116

Seth, A. C., Dalcanton, J. J., Hodge, P. W., \& Debattista, V. P. 2006, AJ, 132, 2539

Spinnato, P. F., Fellhauer, M., \& Portegies Zwart, S. F. 2003, MNRAS, 344, 22

Strigari, L. E., Bullock, J. S., Kaplinghat, M., Simon, J. D., Geha, M., Willman, B., \& Walker, M. G. 2008, Nature, 454, 1096

Tacconi, L. J., et al. 2010, Nature, 463, 781

Tanaka, H., Takeuchi, T., \& Ward, W. R. 2002, ApJ, 565, 1257

Tremaine, S. D., Ostriker, J. P., \& Spitzer, L., Jr. 1975, ApJ, 196, 407

Velazquez, H., \& White, S. D. M. 1999, MNRAS, 304, 254

Wahde, M., Donner, K. J., \& Sundelius, B. 1996, MNRAS, 281, 1165

Walcher, C. J., Böker, T., Charlot, S., Ho, L. C., Rix, H.-W., Rossa, J., Shields, J. C., \& van der Marel, R. P. 2006, ApJ, 649, 692

Walcher, C. J., et al. 2005, ApJ, 618, 237

Wandel, A. 1999, ApJ, 519, L39

Wehner, E. H., \& Harris, W. E. 2006, ApJ, 644, L17

Weinzirl, T., Jogee, S., Khochfar, S., Burkert, A., \& Kormendy, J. 2009, ApJ, 696, 411

Whitmore, B. C., Chandar, R., \& Fall, S. M. 2007, AJ, 133, 1067

Wyse, R. F. G. 2010, Astron. Nachr., 331, 526

Zavala, J., Okamoto, T., \& Frenk, C. S. 2008, MNRAS, 387, 364

Zhang, Q., \& Fall, S. M. 1999, ApJ, 527, L81

Zhao, D. H., Mo, H. J., Jing, Y. P., \& Boumlrner, G. 2003, MNRAS, 339, 12 